\title{
Aortic diameter assessment by cardiovascular magnetic resonance: do we really need contrast enhanced images?
}

\author{
Irene Ferrari ${ }^{1}$, Nerejda Shehu ${ }^{1}$, Naira Mkrtchyan ${ }^{1}$, Heiner Latus ${ }^{1}$, Heiko Stern ${ }^{1}$, Stefan Martinoff ${ }^{2}$, \\ Peter Ewert ${ }^{1}$, Christian Meierhofer ${ }^{1}$ \\ ${ }^{1}$ Pediatric Cardiology and Congenital Heart Disease, German Heart Center Munich, Technical University Munich, Munich, Germany; ${ }^{2}$ Radiology, \\ German Heart Center Munich, Technical University Munich, Munich, Germany \\ Contributions: (I) Conception and Design: I Ferrari, H Stern, C Meierhofer; (II) Administrative Support: None; (III) Provision of study materials or \\ patients: I Ferrari, P Ewert, C Meierhofer; (IV) Collection and assembly of data: I Ferrari, N Shehu, N Mkrtchyan, C Meierhofer; (V) Data analysis \\ and interpretation: I Ferrari, N Mkrtchyan, H Stern, C Meierhofer; (VI) Manuscript writing: All Authors; (VII) Final approval of manuscript: All \\ Authors. \\ Correspondence to: Irene Ferrari, MD. German Heart Centre Munich, Lazarettstr. 36, 80636 Munich, Germany. Email: irene.ferrari@hotmail.com.
}

Background: Cardiovascular magnetic resonance (CMR) is widely used for aortic diameter assessment but there is no consensus on the sequence or cardiac cycle phase in which the measures should be taken. The most used sequence is contrast-enhanced-magnetic-resonance-angiography (angiography), usually nonECG-triggered. An alternative is a navigated 3D-whole-heart-steady-state-free-precession sequence which is contrast-free and breath- and ECG-gated (mostly diastolic gating), producing very sharp anatomical rendering. Nonetheless, its routine use has not yet spread. Our aim was evaluating aortic diameters by a systolic-gated $3 \mathrm{D}$ and put additional effort in the validation of diastolic-gated $3 \mathrm{D}$ as alternative to angiography.

Methods: We retrospectively analysed 30 patients scheduled for routine Angiography. We measured the aorta at 9 standard positions by three different sequences (angiography, 3D-diastole and 3D-systole) and compared the diameters obtained by calculating the differences and by paired $t$-test analysis.

Results: Diameters by 3D-systole were larger than by $3 \mathrm{D}$-diastole and angiography $(\mathrm{P}<0.01)$. In the ascending aorta we found the maximal differences between systole and diastole and between systole and angiography which were $1.7 \pm \mathrm{SD} 1.02 \mathrm{~mm}$ and $1.5 \pm \mathrm{SD} 1.07 \mathrm{~mm}$ respectively. There was no significant difference between diastolic and angiography measurements (mean difference $0.2 \pm$ SD $0.16 \mathrm{~mm}, \mathrm{P}$ not significant).

Conclusions: Our results support the use of navigated 3D-whole-heart CMR to evaluate aortic diameters. Systolic-gated 3D produces larger diameter, especially in the ascending aorta.

Keywords: Aortic diameter assessment; cardiovascular magnetic resonance (CMR); aortic wall disease; Marfan syndrome

Submitted Oct 21, 2020. Accepted for publication Feb 01, 2021.

doi: $10.21037 / \mathrm{cdt}-20-868$

View this article at: http://dx.doi.org/10.21037/cdt-20-868

^ORCID: 0000-0001-9005-2856. 


\section{Introduction}

Cardiovascular magnetic resonance (CMR) is widely used for aortic diameter assessment but there is to date no consensus on the sequence, cardiac cycle phase and modality in which the measures should be taken $(1,2)$. One of the most used tools is contrast-enhanced-MR angiography (ceMRA). Though having gained broad acceptance, this sequence carries some major limitations: first, the use of contrast medium is not recommended or even contraindicated in patients with impaired renal function or allergic diathesis. Second, repeated contrast administrations result in brain deposition of gadolinium, whose long-term effect is yet to be understood (3); this issue gains relevance especially for patients who need lifelong annual or even closer follow-up examinations, such is the case in Marfan, Ehlers-Danlos (EDS), Loeys-Dietz syndrome as well as many acquired aortic diseases. Lastly, ceMRA is usually non-ECG-gated, therefore producing images with a certain amount of blurring, mostly intense at the aortic root level, where optimal anatomic evaluation and reproducibility of the diameter assessment is particularly crucial. A good alternative to ceMRA is an ECG-gated navigatortriggered 3D-SSFP-whole-heart sequence (3D), which can produce a whole chest scan in a contrast-free, ECGgated and breath-gated technique, with consequent very sharp anatomical rendering of the whole vessel, including the aortic root. The $3 \mathrm{D}$ can be gated both to systole and diastole, although in the clinical practice, diastolic trigger is almost exclusively used because of less movement artefact and better image quality. There are already small studies that show non-inferiority of this sequence compared to ceMRA as mean to assess aortic diameter as well as thoracic aortic disease (4-6); nonetheless, the routine use of $3 \mathrm{D}$ for evaluation of aortic diameters has not yet spread, and ceMRA remains the sequence of choice in the main works used as reference for normal CMR-based aortic diameters both in adults and children (7). Moreover, so far there are no studies that systematically evaluate the $3 \mathrm{D}$ sequence during systole; systolic-gated images may play an important role in case of arrhythmias in which diastolic triggering results in suboptimal image quality as well as in aorticwall pathologies in which the vessel may dilate significantly during systole. Our purpose was to verify if the $3 \mathrm{D}$ technique could be a valid alternative to ceMRA in order to promote its preferential use in the clinical routine for aortic diameter assessment; secondly, we wanted to test a systolic-gated 3D and provide quantification of their relation to diastolic-gated 3D and ceMRA diameters. We present the following article

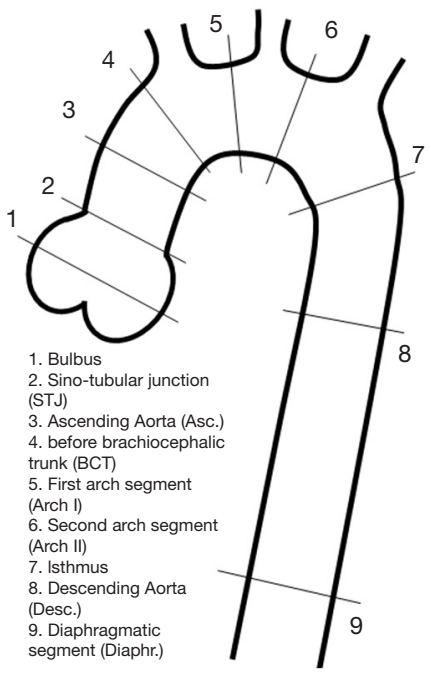

Figure 1 Anatomic locations of aorta measurements.

in accordance with the MDAR reporting checklist (available at http://dx.doi.org/10.21037/cdt-20-868).

\section{Methods}

We retrospectively analyzed $30 \mathrm{CMR}$-examinations in patients scheduled for routine CMR for follow-up of various congenital heart disease; none of them had aortic disease as primary indication for CMR. CMR was performed with a $1.5 \mathrm{~T}$ scanner (Avanto, Siemens Healthcare, Erlangen, Germany). Ungated ceMRA was performed during breath-hold as previously described $(8,9)$; 3D-whole heart CMR was performed twice, in systole and late diastole during free breathing with a motionadaptive navigator technique, as previously described $(8,10)$. Data analysis was performed offline using commercially available software (Argus ${ }^{\circledR}$, Siemens Healthcare, Erlangen, Germany). The aortic diameters were measured at 9 standard positions from the root to the descending aorta as shown in Figure 1. Three diameters were taken at the level of the aortic bulbus (cusp-cusp-cusp), while two diameters were taken at all other levels; the mean of the values was taken as representative diameter; diameters where taken from the inner-edge to the inner-edge of the vessel. We compared diameters at each level in systole, diastole and ceMRA by paired $t$-test. We described the absolute difference of the diameters taken by the three different sequences. We performed intra- and inter-observer statistics by Bland-Altman analysis. For interoperator variability, a second operator repeated the measures of 7 patients in three of the aortic positions (bulbus, ascending aorta, descending aorta). For intra-operator variability the main operator repeated the measures of 7 patients in five of the 

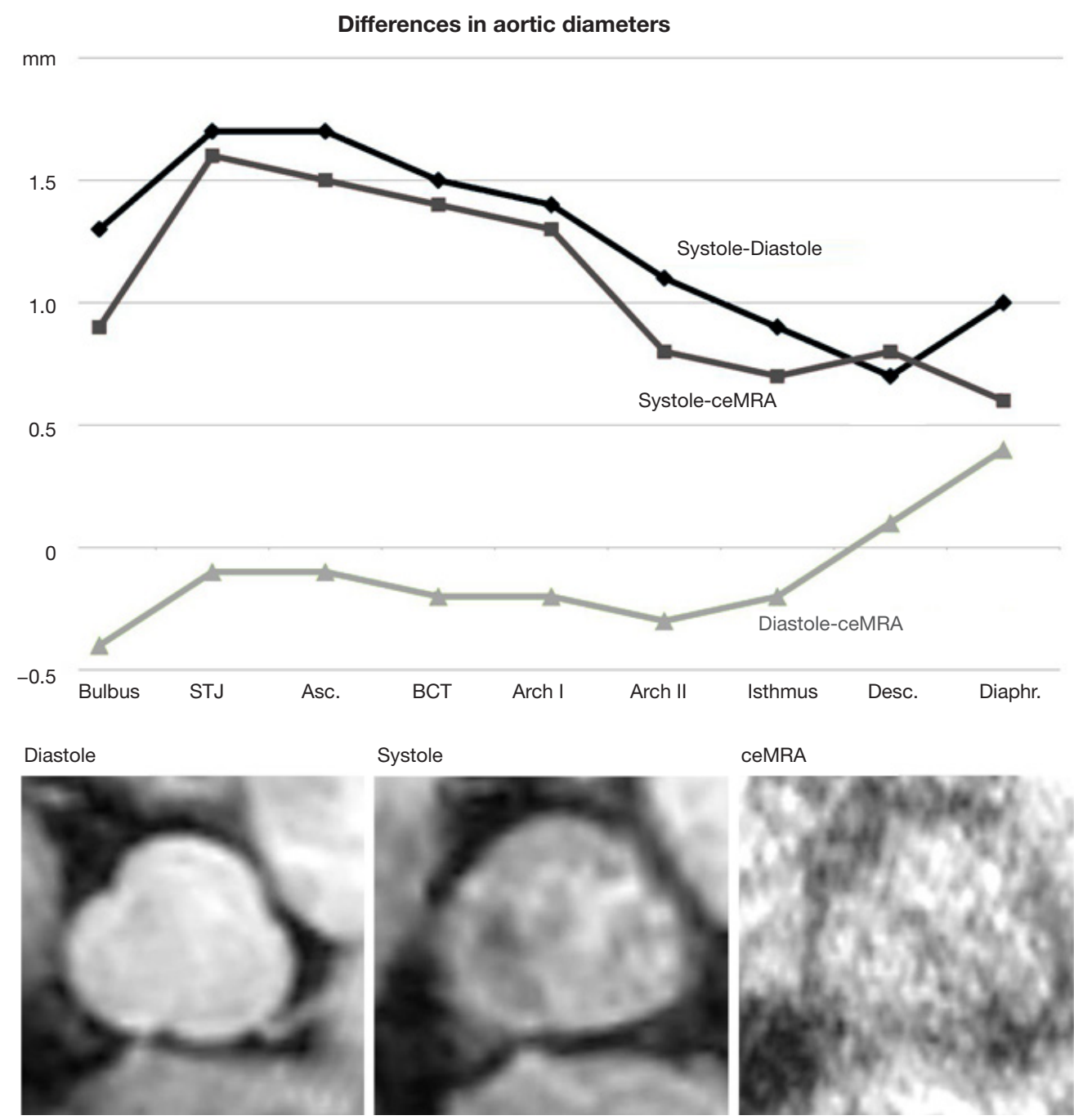

CeMRA

Figure 2 Graphic with numeric absolute difference $(\mathrm{mm})$ between the three different sequences through which the aorta was assessed. Systolic diameters were larger than diameters assessed by the two other methods, mostly at the level of the ascending aorta. Below the graphic, the three figures demonstrate the anatomical rendering of the aortic root by the three different sequences; blurring due to movement artefacts is evident by ceMRA; better sharpness is obtained by 3D whole heart CMR, especially using diastolic gating. CMR, cardiovascular magnetic resonance; ceMRA, contrast-enhanced-MR angiography.

aortic positions (bulbus, ascending aorta, second aortic arch segment, isthmus, descending aorta). The study was approved by the Institutional Review Board (Ethical Committee of the Technical University of Munich; study registration number $1 / 15 \mathrm{~s})$. The study was conducted in accordance with the Declaration of Helsinki (as revised in 2013). Since study design was retrospective, no informed consent was needed.

\section{Results}

Diameters obtained by 3D systole were significantly larger than those by $3 \mathrm{D}$ diastole and by ceMRA $(\mathrm{P}<0.001)$. There was no significant difference between diameters by $3 \mathrm{D}$ diastole and by ceMRA ( $\mathrm{P}$ always $>0.1$; overall mean difference $0.2 \pm$ SD $0.16 \mathrm{~mm}$, not significant). In the ascending aorta the mean differences between systolic and diastolic diameters and between systolic and ceMRA diameters were $1.7 \pm$ SD $1.02 \mathrm{~mm}$ and $1.5 \pm \mathrm{SD} 1.07 \mathrm{~mm}$, respectively. The differences gradually decreased to a minimum of $0.7 \pm$ SD $0.91 \mathrm{~mm}$ and $0.8 \pm$ SD $0.69 \mathrm{~mm}$ in the descending aorta (Figure 2). For inter-operator statistics, results were as follows: $95 \%$ limits of agreement: -3.732 to 
Table 1 Aortic diameters at all levels by different sequences

\begin{tabular}{lccc}
\hline \multirow{2}{*}{ Aortic level } & & Aortic diameter $(\mathrm{mm})$ & ceMRA \\
\cline { 2 - 4 } Bulbus & 3D systole & 3D diastole & $34.7 \pm 5.1$ \\
STJ & $35.2 \pm 5.4$ & $33.9 \pm 5.6$ & $30.3 \pm 5.4$ \\
Asc. & $31.8 \pm 5.3$ & $30.1 \pm 5.4$ & $29.8 \pm 4.9$ \\
BCT & $31.3 \pm 5.2$ & $29.7 \pm 5.2$ & $26.8 \pm 4.4$ \\
Arch I & $28.2 \pm 4.6$ & $26.7 \pm 4.6$ & $25.9 \pm 4.2$ \\
Arch II & $27.2 \pm 4.0$ & $25.9 \pm 4.3$ & $23.0 \pm 3.5$ \\
Isthmus & $23.8 \pm 3.8$ & $22.7 \pm 3.9$ & $18.9 \pm 3.5$ \\
Desc. & $19.7 \pm 3.8$ & $18.7 \pm 3.7$ & $16.5 \pm 2.7$ \\
Diaphr. & $17.7 \pm 2.9$ & $14.7 \pm 2.6$ & $16.6 \pm 2.7$ \\
\hline
\end{tabular}

Diameters are presented as mean \pm standard deviation (SD); each value is the mathematical mean of three diameters at the level of the Bulbus (cusp-cusp-cusp method) and of two diameters (major diameter, minor diameter) at all other levels. STJ, sino-tubular junction; Asc., ascending aorta; BCT, before brachiocephalic trunk; Arch I, first arch segment; Arch II, second arch segment; Desc., descending aorta; Diaphr., diaphragmatic segment.

3.403 (bias -0.1645 , SD of bias 1.820 ). For intra-operator statistics, results were as follows: $95 \%$ limits of agreement: -1.941 to 1.854 (bias -0.04356 : SD of bias 0.9680 ). All mean and median diameters at each level by each sequence are reported in Table 1 .

\section{Discussion}

According to our results, navigated 3D whole-heart CMR with diastolic trigger produces diameters that are not significantly different from diameters taken by ceMRA; the sequence is contrast-free and produces sharper images, especially at the aortic root level, which is often a crucial spot to evaluate. This finding is very important to consider when performing long follow-ups with serial examinations over the years because it implies sparing of repeated contrast medium administration (e.g., arteriopathies such as Marfan, EDS, Loeys-Dietz, dilatation of ascending aorta in aortic stenosis/bicuspid aortic valve); moreover, sparing of contrast administration may be crucial in the pediatric population in which the need of an intravenous line is not free from impact for the subsequent collaboration of the patients during the CMR-acquisition.

This work also contains a whole set of measurements of the aorta by navigated 3D whole-heart CMR in systole and provide quantification of their relation to diastolic and ceMRA diameters; we could quantify the amount of dilatation of the vessel during systole, showing how this difference, though small, is statistically relevant; this dilation may be of greater relevance in patients with aortic wall disease (as was not the case in our cohort), in which altered histological structure of the aortic wall can cause a more pronounced systolic dilation of the vessel. In our cohort, the difference between $3 \mathrm{D}$ systole and the two other sequences (3D diastole and ceMRA) was bigger than the interoperator difference, which demonstrates again the importance of the type of sequence used. Best agreement in the interoperator statistics, was found for the measures taken by 3D-SSFP in diastole, which underlines how the better quality of the images makes this sequence the more reliable for serial evaluation of aortic diameters; the use of this sequence implies sparing of routine use of contrast medium, which, in our opinion, should be reserved to cases where acute or subacute aortic wall disease is suspected. Our results point out the need for a more detailed description on how to evaluate aortic diameters by different imaging modalities, currently lacking in the guidelines. Method of measurement (inner vs. outer vs. leading edge), type of sequence and phase of the cardiac cycle should be specified. Moreover, guidelines should address the problem of different way of sizing the aortic bulbus (cusp-cusp vs. cusp-commissure). This should help to objectify the actual dilation of the vessel, avoiding errors related to inter- and intraoperator difference, as well as differences related to method of 
measurement. Study limitations are the retrospective design and the small study cohort; moreover, our analysis could gain additional significance when performed in a cohort of patients with aortic wall disease.

\section{Conclusions}

Our results support the use of navigated 3D whole-heart CMR to evaluate aortic diameters; the use of ceMRA in our opinion has to be reserved for those cases in which acute or subacute aortic wall disease is suspected; this attitude is coherent with the clinical routine at our institution, where only a very small number of patients evaluated for aortic wall disease receive contrast medium. Systolic-gated 3D produce larger diameter, especially in the ascending aorta; this should be considered in cases that have a borderline indication to surgery, where a few millimetres can make a difference.

\section{Acknowledgments}

Funding: None.

\section{Footnote}

Provenance and Peer Review: This article was commissioned by the Guest Editors (Yskert von Kodolitsch, Harald Kaemmerer, Koichiro Niwa) for the series "Current Management Aspects in Adult Congenital Heart Disease (ACHD): Part IV" published in Cardiovascular Diagnosis and Therapy. The article has undergone external peer review.

Reporting Checklist: The authors have completed the MDAR reporting checklist. Available at http://dx.doi.org/10.21037/ cdt-20-868

Data Sharing Statement: Available at http://dx.doi. org/10.21037/cdt-20-868

Conflicts of Interest: All authors have completed the ICMJE uniform disclosure form (available at http:// dx.doi.org/10.21037/cdt-20-868). The series "Current Management Aspects in Adult Congenital Heart Disease (ACHD): Part IV" was commissioned by the editorial office without any funding or sponsorship. The authors have no other conflicts of interest to declare.

Ethical Statement: The authors are accountable for all aspects of the work in ensuring that questions related to the accuracy or integrity of any part of the work are appropriately investigated and resolved. The study was approved by the Institutional Review Board (Ethical Committee of the Technical University of Munich; study registration number $1 / 15 \mathrm{~s}$ ). The study was conducted in accordance with the Declaration of Helsinki (as revised in 2013). Since study design was retrospective, no informed consent was needed.

Open Access Statement: This is an Open Access article distributed in accordance with the Creative Commons Attribution-NonCommercial-NoDerivs 4.0 International License (CC BY-NC-ND 4.0), which permits the noncommercial replication and distribution of the article with the strict proviso that no changes or edits are made and the original work is properly cited (including links to both the formal publication through the relevant DOI and the license). See: https://creativecommons.org/licenses/by-nc-nd/4.0/.

\section{References}

1. Erbel R, Aboyans V, Boileau C, et al. Corrigendum to: 2014 ESC Guidelines on the diagnosis and treatment of aortic diseases. Eur Heart J 2015;36:2779.

2. Hiratzka LF, Bakris GL, Beckman JA, et al. 2010 ACCF/AHA/AATS/ACR/ASA/SCA/SCAI/SIR/STS/ SVM guidelines for the diagnosis and management of patients with Thoracic Aortic Disease: a report of the American College of Cardiology Foundation/American Heart Association Task Force on Practice Guidelines, American Association for Thoracic Surgery, American College of Radiology, American Stroke Association, Society of Cardiovascular Anesthesiologists, Society for Cardiovascular Angiography and Interventions, Society of Interventional Radiology, Society of Thoracic Surgeons, and Society for Vascular Medicine. Circulation 2010;121:e266-369.

3. Gulani V, Calamante F, Shellock FG, et al. Gadolinium deposition in the brain: summary of evidence and recommendations. Lancet Neurol 2017;16:564-70.

4. Potthast S, Mitsumori L, Stanescu LA, et al. Measuring aortic diameter with different MR techniques: comparison of three-dimensional (3D) navigated steady-state freeprecession (SSFP), 3D contrast-enhanced magnetic resonance angiography (CE-MRA), 2D T2 black blood, and 2D cine SSFP. J Magn Reson Imaging 2010;31:177-84.

5. Veldhoen S, Behzadi C, Lenz A, et al. Non-contrast MR 
angiography at 1.5 Tesla for aortic monitoring in Marfan patients after aortic root surgery. J Cardiovasc Magn Reson 2017;19:82.

6. Krishnam MS, Tomasian A, Malik S, et al. Image quality and diagnostic accuracy of unenhanced SSFP MR angiography compared with conventional contrastenhanced MR angiography for the assessment of thoracic aortic diseases. Eur Radiol 2010;20:1311-20.

7. Kawel-Boehm N, Maceira A, Valsangiacomo-Buechel ER, et al. Normal values for cardiovascular magnetic resonance in adults and children. J Cardiovasc Magn Reson 2015;17:29.

8. Fratz S, Chung T, Greil GF, et al. Guidelines and

Cite this article as: Ferrari I, Shehu N, Mkrtchyan N, Latus H, Stern H, Martinoff S, Ewert P, Meierhofer C. Aortic diameter assessment by cardiovascular magnetic resonance: do we really need contrast enhanced images? Cardiovasc Diagn Ther 2021;11(6):1389-1394. doi: 10.21037/cdt-20-868 protocols for cardiovascular magnetic resonance in children and adults with congenital heart disease: SCMR expert consensus group on congenital heart disease. J Cardiovasc Magn Reson 2013;15:51.

9. Kondo C, Takada K, Yokoyama U, et al. Comparison of three-dimensional contrast-enhanced magnetic resonance angiography and axial radiographic angiography for diagnosing congenital stenoses in small pulmonary arteries. Am J Cardiol 2001;87:420-4.

10. Martirosian P, Greil GF, Fenchel M, et al. Optimization of blood-myocardial contrast in 3D true FISP cardiac imaging at 1.5 T. Magn Reson Med 2007;57:213-9. 